\title{
Cardiovascular magnetic resonance detects microvascular dysfunction in a mouse model of hypertrophic cardiomyopathy
}

Min-Chi Ku ${ }^{1,2^{*}} \mathbb{D}$, Frank Kober ${ }^{3}$, Yi-Ching Lai ${ }^{1}$, Andreas Pohlmann ${ }^{1}$, Fatimunnisa Qadri', Michael Bader ${ }^{2,4}$, Lucie Carrier ${ }^{5,6}$ and Thoralf Niendorf $1,2,7$

\begin{abstract}
Background: Hypertrophic cardiomyopathy (HCM) related myocardial vascular remodelling may lead to the reduction of myocardial blood supply and a subsequent progressive loss of cardiac function. This process has been difficult to observe and thus their connection remains unclear. Here we used non-invasive myocardial blood flow sensitive CMR to show an impairment of resting myocardial perfusion in a mouse model of naturally occurring HCM.
\end{abstract}

Methods: We used a mouse model (DBA/2 J; D2 mouse strain) that spontaneously carries variants in the two most susceptible HCM genes-Mybpc3 and Myh7 and bears the key features of human HCM. The C57BL/6 J (B6) was used as a reference strain. Mice with either B6 or D2 backgrounds (male: $n=4$, female: $n=4$ ) underwent cine-CMR for functional assessment at 9.4 T. Left ventricular (LV) wall thickness was measured in end diastolic phase by cine-CMR. Quantitative myocardial perfusion maps (male: $n=5$, female: $n=5$ in each group) were acquired from arterial spin labelling (cine ASL-CMR) at rest. Myocardial perfusion values were measured by delineating different regions of interest based on the LV segmentation model in the mid ventricle of the LV myocardium. Directly after the CMR, the mouse hearts were removed for histological assessments to confirm the incidence of myocardial interstitial fibrosis $(n=8$ in each group) and small vessel remodelling such as vessel density ( $n=6$ in each group) and perivascular fibrosis ( $n=8$ in each group).

Results: LV hypertrophy was more pronounced in D2 than in B6 mice (male: D2 LV wall thickness $=1.3 \pm 0.1 \mathrm{~mm}$ vs B6 LV wall thickness $=1.0 \pm 0.0 \mathrm{~mm}, p<0.001$; female: $\mathrm{D} 2 \mathrm{LV}$ wall thickness $=1.0 \pm 0.1 \mathrm{~mm}$ vs B6 LV wall thickness $=0.8 \pm 0.1 \mathrm{~mm}, p<0.01$ ). The resting global myocardial perfusion (myocardial blood flow; MBF) was lower in D2 than in $\mathrm{B} 6$ mice (end-diastole: $\mathrm{D} 2 \mathrm{MBF}_{\text {global }}=7.5 \pm 0.6 \mathrm{vs} \mathrm{B6} \mathrm{MBF}_{\text {global }}=9.3 \pm 1.6 \mathrm{ml} / \mathrm{g} / \mathrm{min}, p<0.05$; end-systole: D2 $\left.\mathrm{MBF}_{\text {global }}=6.6 \pm 0.8 \mathrm{vs} \mathrm{B6} \mathrm{MBF}_{\text {global }}=8.2 \pm 2.6 \mathrm{ml} / \mathrm{g} / \mathrm{min}, p<0.01\right)$. This myocardial microvascular dysfunction was observed and associated with a reduction in regional MBF, mainly in the interventricular septal and inferior areas of the myocardium. Immunofluorescence revealed a lower number of vessel densities in D2 than in B6 (D2 capillary $=31.0 \pm 3.8 \%$ vs B6 capillary $=40.7 \pm 4.6 \%, p<0.05)$. Myocardial collagen volume fraction (CVF) was significantly higher in D2 LV versus B6 LV mice (D2 CVF $=3.7 \pm 1.4 \%$ vs $B 6 C V F=1.7 \pm 0.7 \%, p<0.01$ ). Furthermore, a higher ratio of perivascular fibrosis (PFR) was found in D2 than in B6 mice (D2 PFR $=2.3 \pm 1.0 \%, \mathrm{~B} 6 \mathrm{PFR}=0.8 \pm 0.4 \%, p<0.01$ ).

\footnotetext{
${ }^{*}$ Correspondence: min-chi.ku@mdc-berlin.de

${ }^{1}$ Berlin Ultrahigh Field Facility (B.U.F.F.), Max Delbrück Center for Molecular

Medicine in the Helmholtz Association (MDC), Robert-Rössle Strasse 10,

13125 Berlin, Germany

Full list of author information is available at the end of the article
}

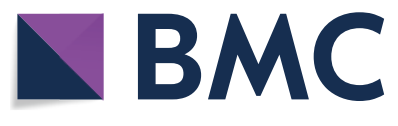
permits use, sharing, adaptation, distribution and reproduction in any medium or format, as long as you give appropriate credit to the original author(s) and the source, provide a link to the Creative Commons licence, and indicate if changes were made. The images or other third party material in this article are included in the article's Creative Commons licence, unless indicated otherwise in a credit line to the material. If material is not included in the article's Creative Commons licence and your intended use is not permitted by statutory regulation or exceeds the permitted use, you will need to obtain permission directly from the copyright holder. To view a copy of this licence, visit http://creativecommons.org/licenses/by/4.0/. The Creative Commons Public Domain Dedication waiver (http://creativeco mmons.org/publicdomain/zero/1.0/) applies to the data made available in this article, unless otherwise stated in a credit line to the data. 
Conclusions: Our work describes an imaging marker using cine ASL-CMR with a potential to monitor vascular and myocardial remodelling in HCM.

Keywords: Hypertrophic cardiomyopathy (HCM), Myocardial remodelling, Microvascular dysfunction, Perfusion, Myocardial blood flow (MBF), Cardiac MRI (CMR), Arterial spin labelling (ASL)

\section{Background}

Hypertrophic cardiomyopathy (HCM) is the most prevalent cardiac genetic disease with an estimated population prevalence of HCM ranging from 1:200 to 1:500 individuals $[1,2]$ and mainly caused by distinct genetic variants in sarcomeric proteins. The major clinical feature of HCM is left ventricular (LV) hypertrophy (LVH), which is often asymmetric and preferentially affects the interventricular septum, in the absence of any other conditions that could induce LVH, such as environmental factors (stress or physical activities), hypertension, or aortic stenosis [9]. Sudden cardiac death can be the first manifestation of HCM, particularly in young [3, 4] and asymptomatic patients [5, 6]. The key sarcomere genes [7] most frequently affected genes in HCM are the cardiac myosin-binding protein $\mathrm{C}$ (MYBPC3) [8] and $\beta$-myosinheavy chain (MYH7) [9]. Aberrant proteins trigger myocardial tissue remodelling which is a complex process of transcriptional, signaling, structural, electrophysiological, and functional events occurring within the cardiac myocyte and myocardium [10]. The myocardial tissue remodelling contributes to small vessel disease (namely vascular remodelling), cardiac hypertrophy, myocyte disarray, myocardial fibrosis and ultimately compromise the cardiac function $[11,12]$. While the early detection of myocardial remodelling is a key to effective disease management [13], at present no in vivo imaging markers of dynamic changes of microvasculature have been found [14]. CMR can detect ventricular wall hypertrophy and fibrosis progression, two main features that are useful in predicting $\mathrm{HCM}$ prognoses [15], but monitoring those morphological changes on the macroscopic and mesoscopic scales cannot detect subclinical microvascular dysfunctions, which potentially might be more sensitive markers of disease progression [16]. Here we focused on the remodelling of small vessels and microvascular dysfunction in the myocardium, which may lead to a reduction of myocardial blood supply and a subsequent progressive loss or deterioration of cardiac function. In response to varying physiological or pathological conditions and functional demands, blood vessels are continuously adapting their structural change [12].

Microvascular dysfunction in HCM patients has been proposed as a strong predictor of clinical outcomes and mortality risk [17], but the relationship between this and myocardial remodelling is poorly understood.
The small vessels of the microvascular network supply the myocardium with oxygen to maintain functional cardiac tissue integrity [18]. Myocardial wall thickening connected to pressure overload is thought to play a role in impairing coronary inflow and trigger a progressive ventricular dysfunction [19]. Hypertrophy-induced deficits in myocardial perfusion and micro-circulation might also trigger the pathological deposition of collagen in the myocardium. This reduces myocardial blood flow and might be the key determinant of subsequent heart failure [20]. This suggests that monitoring changes in micro-perfusion might make an effective early indicator to diagnose the disease and have the chance to provide early procedures that improve blood flow.

Local myocardial blood volume and blood flow are important indicators of changes in micro-perfusion [21]. Recent work has investigated the relationship between hypertrophy and the myocardial microvascular network in HCM [22]. In these studies, the fractional tissue blood volume per cardiac tissue volume (Bvf), defined as the volume in the microvascular network, was directly probed by Bvf-sensitive CMR using blood oxygenation-weighted imaging contrast [22]. Myocardial regions with reduced Bvf were highly correlated with ventricular wall thickening $[22,23]$. Yet the factors responsible for the reduction of microvascular network volume remain unknown.

We hypothesize that vascular dysregulation caused by hypertrophy or excessive collagen deposits may contribute to the microstructural changes in HCM and cause the reduction. Using a mouse model that closely reflects human HCM, we quantified the amount of blood delivered to myocardial tissue per unit time using myocardial blood flow (MBF) CMR based on the labelling of arterial proton spins [24, 25]. Unlike widely used methods based on contrast agent enhanced first pass perfusion CMR, arterial spin labelling (ASL)-CMR does not require an injection of exogenous contrast material, and thus can be repeated [26]. Employing this approach, the goal was to find any sign of small vessel change indicated by myocardial perfusion and then correlate the changes detectable by CMR with microstructural histology in the murine heart - something that is difficult to do in human patients. For this purpose MBF, capillary density, perivascular fibrosis and interstitial fibrosis were determined. 


\section{Methods}

Mouse model carries HCM gene variants

All animal studies were approved by the Berlin State review board at the "Landesamt für Gesundheit und Soziales (LAGeSo; State Office for Health and Social Affairs Berlin)". Genetic variants that potentially cause myocardial functional changes were previously reported [27]. We used a mouse model (DBA/2J; D2 mouse strain) that spontaneously carries variants in the two most susceptible HCM genes-Mybpc3 and Myh7 and bears the key features of human HCM [28]. The C57BL/6J (B6) strain was used as reference. Mice were handled according to the LAGeSo and internal (MDC) rules and regulations. In total, 36 eight-month-old mice were used (4 male and 4 female mice with B6 or D2 backgrounds were used for cardiac functional assessment and 5 male and 5 female mice with B6 or D2 were used for ASL-CMR). The experimental design is illustrated in Fig. 1a.

\section{Cardiovascular magnetic resonance imaging Physiological control}

All in vivo CMR measurements were performed on a 9.4 T small animal CMR system (Biospec 94/20, Bruker Biospin, Germany). Anesthesia of mice was induced using 3\% isoflurane (CP-Pharma, Germany) in $500 \mathrm{~mL} /$ min medical air and $500 \mathrm{~mL} / \mathrm{min}$ oxygen and was maintained at 0.5 to $1.0 \%$ isoflurane after the induction. During CMR examinations, it was important to maintain hemodynamic stability to avoid confounding interference with the imaging results. During cine ASL-CMR scans, oxygen was given at a dose of at least $800 \mathrm{~mL} / \mathrm{min}$ to avoid hypoxia. Core body temperature was maintained at $36 \pm 0.5^{\circ} \mathrm{C}$ using a heated water tubing system. Heart rates, respiration rates and core body temperature were closely monitored using a remote monitoring system (Model 1025, SA Instruments Inc., Stony Brook, New York, USA).

\section{Cardiac physiological assessments}

For the high-fidelity approaches for CMR in mice, image acquisition was conducted using a cryogenically-cooled radiofrequency (RF) transceiver antenna (CryoProbe, Bruker Biospin). To obtain a stack of cardiac short axis (SAX) views covering the whole mouse heart, 9-10 slices were consecutively acquired using a self-gated bright-blood cine (IntraGate-FLASH, TR/ $\mathrm{TE}=8.5 / 1.58 \mathrm{~ms}, \mathrm{FA}=20^{\circ}$, receiver bandwidth $=98 \mathrm{kHz}$, FOV $=11 \times 22 \mathrm{~mm}^{2}$, matrix size $=192 \times 384$, slice thickness $=0.8 \mathrm{~mm}$, with a temporal resolution of 16 images per cardiac cycle [29]. Cardiac function assessment was performed using cvi42 (Circle Cardiovascular Imaging, Calgary, Alberta, Canada) and analyzed on a

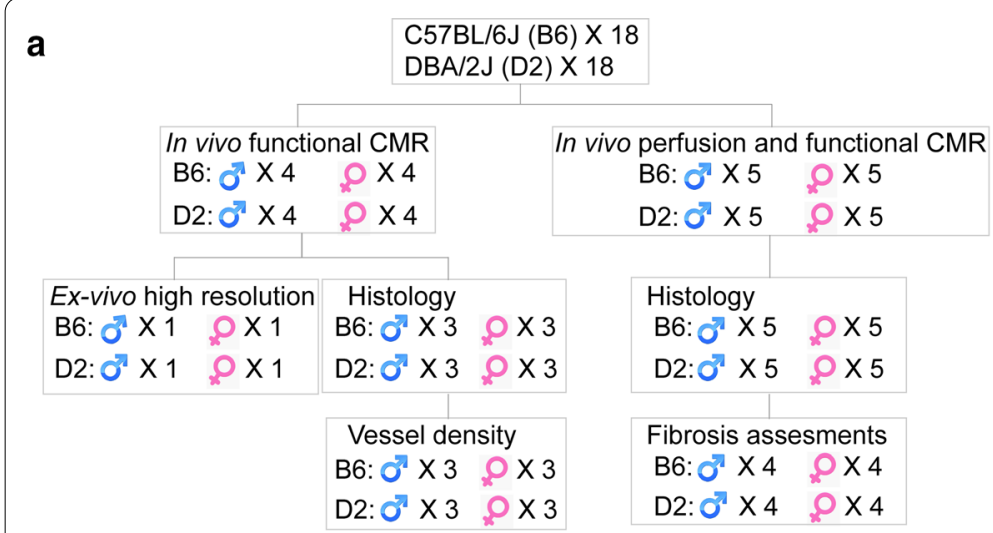

b Spin labelling

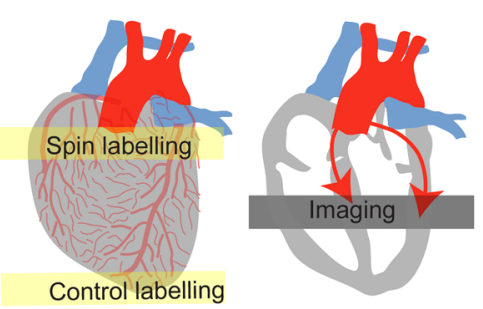

Control labelling
C Myocardial perfusion maps
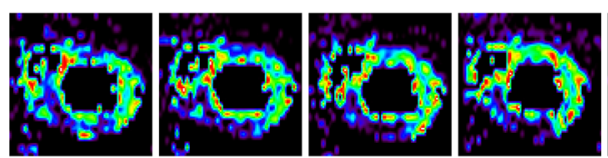

diastole
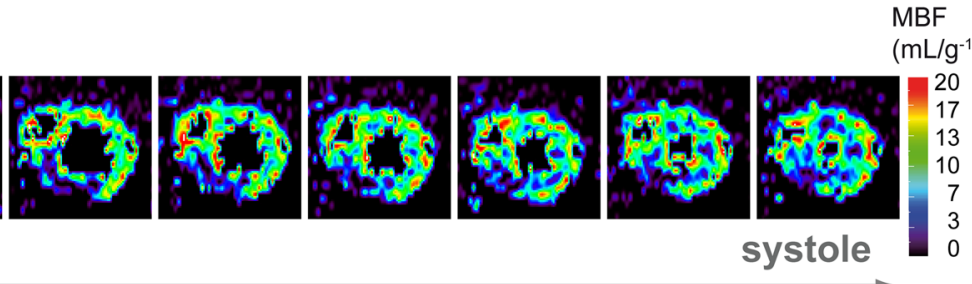

Fig. 1 a Schematic representation of experimental design. b Schematic illustration of the tagging slice position used for quantifying myocardial blood flow (MBF) with arterial spin labelling (ASL)-cardiovascular magnetic resonance (CMR). By labelling water molecules in the inflowing blood from coronary artery as tracers which then perfused into myocardium, labelled blood signal can be imaged in the mid-left ventricle (LV). c Ten consecutive color coded short axis myocardial perfusion maps from a measurement series over one cardiac cycle 
slice-by-slice basis. Endo- and epi-cardiac borders were manually segmented in end-systole and end-diastole using a stack of SAX cine images. LV ejection fraction (LVEF) and myocardial mass (both in diastole and systole) were calculated. LV wall thickness was measured in end diastolic phase. Six SAX views were used for measuring the maximal wall thickness.

\section{Quantification of myocardial perfusion using cine ASL-CMR (cineASL)}

The cineASL technique used is described in detail elsewhere $[24,30]$. This cineASL approach was previously validated against a classical FAIR Look-Locker GradientEcho technique under rest and stress conditions [30] and built upon a previously published version. The principle of ASL-CMR is to label the inflowing coronary arterial blood as an endogenous diffusible tracer (Fig. 1b). It relies on an electrocardiographically (ECG)-gated cine Fast Low Angle Shot (cine-FLASH) technique repeated over several cardiac cycles for each line of k-space. In a preparation module, spin labelling using dedicated radiofrequency pulses is applied to tag (by inverting the spins) the longitudinal magnetization of coronary blood water protons before it enters the imaging plane in the myocardium [25]. The labelling pulses were produced by replacing one cine readout within the cardiac cycle at the time of valve closure (end systole) by a hyperbolic secant adiabatic inversion pulse. For labelling, a slice-selective inversion slab was placed on the aortic root while another selective inversion slab was placed in the opposite direction below the imaging slice (as control labelling). Two cardiac gated (on ECG) mid-LV SAX cine image series were acquired under labeled and control conditions. For averaging, the acquisition was repeated 35 times over several cardiac cycles for labeled and control conditions without a delay, as described in the original version [30]. Provided that the magnetization of the inflowing blood is the only difference between the control and the label image, a difference map was calculated yielding perfusion-weighted images of the heart for each cardiac cine phase, with the signal intensity being proportional to myocardial perfusion. For quantification of MBF, the acquisitions were done using a $72 \mathrm{~mm}$ inner diameter volume resonator (Bruker Biospin) for uniform RF transmission in conjunction with a 4 channel cardiac RF array (Bruker Biospin) used for signal reception. The following parameters were used for the cineASL technique [24]: $\mathrm{TR} / \mathrm{TE}=7.6 / 1.12 \mathrm{~ms}, \mathrm{FA}=6^{\circ}, \mathrm{FOV}=25 \times 25 \mathrm{~mm}^{2}$, matrix size $=128 \times 64$, slice thickness $=1 \mathrm{~mm}$, temporal resolution within one cardiac cycle $=7.6 \mathrm{~ms}, 35$ averaged cine blocks for both tag and control images. Throughout the ASL measurements, a two lead electrode was used to detect ECG signals of the mouse heart. The ECG trace was monitored with dedicated modules for small animals (SA Instruments Inc.). Image acquisition was gated upon detection of the ECG's R-wave. Quantitative image analysis and calculation of perfusion maps were performed using in-house developed analysis tools built in the Interactive Data Language (IDL). After averaging the steadystate cine repetitions from successive cardiac cycles, regional perfusion was analyzed using a ventricular segmentation model [31]. The border of each myocardial segment was manually delineated for mid-ventricular SAx slices obtained from end-diastolic and end-systolic cardiac phases. Mean MBF were then calculated for regions of interest defined by septal, lateral, anterior, and inferior traced endocardial and epicardial contours which are illustrated in Fig. 4a.

\section{High resolution CMR of ex vivo heart}

Following in vivo CMR, mice were perfused with $4 \%$ PFA. Fixed ex vivo hearts $(\mathrm{n}=2$ from each group for illustration) were imaged with high spatial resolution to check for the presence of myocardial hypertrophy and disarray $\left(\mathrm{RARE}, \mathrm{TR} / \mathrm{TE}=2200 / 40.7 \mathrm{~ms}, \mathrm{FOV}=15 \times 10 \mathrm{~mm}^{2}\right.$, matrix size $=500 \times 336$, slice thickness $=0.3 \mathrm{~mm}$, inplane spatial resolution $=30 \mu \mathrm{m}$ ).

\section{Histological assessment and microscopic image processing}

Directly after the ASL-CMR scans, the mouse hearts were quickly removed from the chest and perfused with ice-cold cardioplegic solution (15 $\mathrm{mM} \mathrm{KCl}$ in PBS). This step arrested the cardiac cycle during diastole. Heart tissue were post fixed in 4\% PFA solution and then dehydrated. Fixed hearts were either cryopreserved in a $30 \%$ sucrose-PBS solution for at least 3 days at $4{ }^{\circ} \mathrm{C}$ or embedded with paraffin. Cryopreserved hearts were then embedded in OCT (Tissue-Tek, Sakura Finetek Germany $\mathrm{GmbH}$, Staufen im Breisgau, Germany), frozen on dry ice, and stored in $-80{ }^{\circ} \mathrm{C}$ until sectioning. For tissue sectioning, $10 \mu \mathrm{m}$ cross-sections of hearts were cryosectioned using a cryostat microtome (Leica CM3050S, Wetzlar, Germany) starting from the apex. Four $\mu \mathrm{m}$ paraffin sections of heart were prepared using a microtome (Thermo Fischer HM355S, Fisher Scientific GmbH, Schwerte, Germany).

\section{Fluorescent microscopy}

For detecting myocardial microvasculature, heart sections were analyzed using immunofluorescence (IF) staining methods. Briefly, $10 \mu \mathrm{m}$ cryo-preserved sections were mounted on glass slides. For blocking the unspecific bindings, tissue sections were treated with a blocking solution containing $10 \%$ normal donkey serum (NDS; Sigma-Aldrich, Taufkirchen, Germany), 4\% fetal bovine serum (BSA; Sigma-Aldrich, Germany), 0.2\% Triton 
$\mathrm{X}-100$ in PBS for $3 \mathrm{~h}$ at room temperature. Heart tissue sections from entire mid-ventricular short-axis sections were then incubated with Alexa Fluor 488 conjugated Isolectin B4 (1:500; Invitrogen) diluted in 0.01 M PBS with $5 \%$ NDS over night at $4{ }^{\circ} \mathrm{C}$. After washing in freshly prepared PBS with $2.5 \%$ NDS, the nuclei were stained with Hoechst 33,342 (Sigma-Aldrich). The presence of fluorescent stained cells was determined by observation on a microscope (BZ 9000 Keyence GmbH, Neu-Isenburg, Germany). Quantifications of Isolectin B4+ vessels were performed in 10 random fields captured under 200 magnifications. All image processing was then performed using Image (National Institutes of Health, Bethesda, Maryland, USA) and Adobe Illustrator (Adobe, San Jose, California, USA). The captured images were carefully screened. Blurred images were excluded from the quantification. For quantification, small vessel density in myocardium was analyzed using ImageJ software plugins (vessel density) in five random microscopic fields in the whole myocardium and expressed as percentage (the number of small vessels per square millimeter tissue).

\section{Light microscopy}

To determine the interstitial collagen (collagen volume fraction; CVF) [32] and perivascular collagen deposit (perivascular fibrosis ratio; PFR) [33] in the mouse heart, cryopreserved or rehydrated heart paraffin sections were stained in Sirius Red solution (detecting collagen) for $1 \mathrm{~h}$ at room temperature. Stained sections were rinsed twice in $1 \%$ acetic acid, dehydrated through serial concentrations of ethanol washes, cleared in xylene, and mounted with a xylene based mounting medium. Extensive washes were performed between each step. Images were taken using a microscope (BZ-9000, Keyence Corporation of America, Itasca, Illinois, USA). CVF was determined as the percent of collagen-stained area/total myocardial area and quantified using ImageJ software. Excluding endocardial and perivascular collagen, the interstitial CVF (\%) was calculated from the area stained by the Sirius red. PFR (\%) was determined from all collagen surrounding an intra-myocardial coronary artery and was calculated as the ratio of the fibrosis area surrounding the vessel to the total vessel area. For each sample, 5 microscopic fields were examined.

\section{Statistical analysis}

All data analyses of the cardiac physiological measurements using CMR and histopathology were performed by reviewers blinded to the mouse genetic makeup. Experimental analysis and statistics were conducted in R studio (R Studio Inc., Boston, USA; http://www.rstudio.com/) or GraphPad Prism 5 (GraphPad Software, La Jolla, California, USA). The graphs were generated by GraphPad
Prism 5. The comparison between B6 and D2 mice in diastolic and systolic cardiac phases were analyzed using one-way ANOVA. The sampling frequency was further tested for normality using Kolmogorov-Smirnov test. A low K-S statistic value represent with a high $\mathrm{p}$-value indicate that data is normally distributed. Statistical difference of MBF by sex or cardiac phases between groups were further tested using factorial analysis of variance (Factorial ANOVA) followed by post-hoc Turkey HSD (Honestly Significant Difference). The Spearman's rank correlation coefficient was used for testing the strength of the correlation between LV thickness and global MBF. Capillary density (\%), CVF (\%) and PFR (\%) from B6 and D2 were compared using $t$-test followed by Mann Whitney test. All data are presented as mean $\pm \mathrm{SD}$. The differences were considered statistically significant at ${ }^{*} P<0.05$; ${ }^{* *} P<0.01$; and ${ }^{* * * *} P<0.001$.

\section{Results \\ Cardiac functional measurements in spontaneous HCM mouse model}

LVH is a key phenotype of HCM. We evaluated the cardiac phenotype in mice carrying natural genetic variants in $M y b p c 3$ and $M y h 7$, which are the two most frequently mutated genes in human HCM [34]. In all mice groups, LV remodelling was not observed in B6 control animals. D2 mice exhibited LVH when compared to B6 reference strain (Fig. 2a). LVH was more pronounced in both male and female D2 mice than in B6 mice (male: D2 LV wall thickness $=1.3 \pm 0.1 \mathrm{~mm}$ vs B6 LV wall thickness $=1.0 \pm 0.03 \mathrm{~mm}, p<0.001$; female: D2 LV wall thickness $=1.0 \pm 0.1 \mathrm{~mm}$ vs B6 LV wall thickness $=0.8 \pm 0.1 \mathrm{~mm}, p<0.01)$ (Fig. 2b). LVEF in D2 mice remained normal and showed no significant difference when compared to B6 (male: D2 LVEF $=70.3 \pm 5.8 \%$ vs $\mathrm{B} 6 \mathrm{LVEF}=73.8 \pm 0.7 \%$; female: $\mathrm{D} 2 \mathrm{LVEF}=71.9 \pm 4.1 \%$, vs B6 LVEF $=68.9 \pm 5.5 \%$; Fig. 2b).

\section{Cine ASL-CMR detection of myocardial perfusion deficit}

We investigated non-invasively the impact of primary genetic variants on myocardial perfusion using MBF sensitive CMR. As previously shown, the cine ASLCMR sequence allows tracking of dynamic MBF changes across the entire cardiac cycle [24], which is necessary to depict any cyclic change in microvascular function in the myocardium. For this purpose we divided the cardiac cycle into 14-17 cardiac phases (depending on the heart rates of individual mice) and allowed depicting the end-diastole and end-systole separately (Fig. 3a, left). No significant differences between males and females were found. In D2 females, a higher end diastolic MBF was noted compared to $\mathrm{D} 2$ males $(\mathrm{P}<0.05)$, but no other significant differences were observed (Fig. $3 a$, right). The 

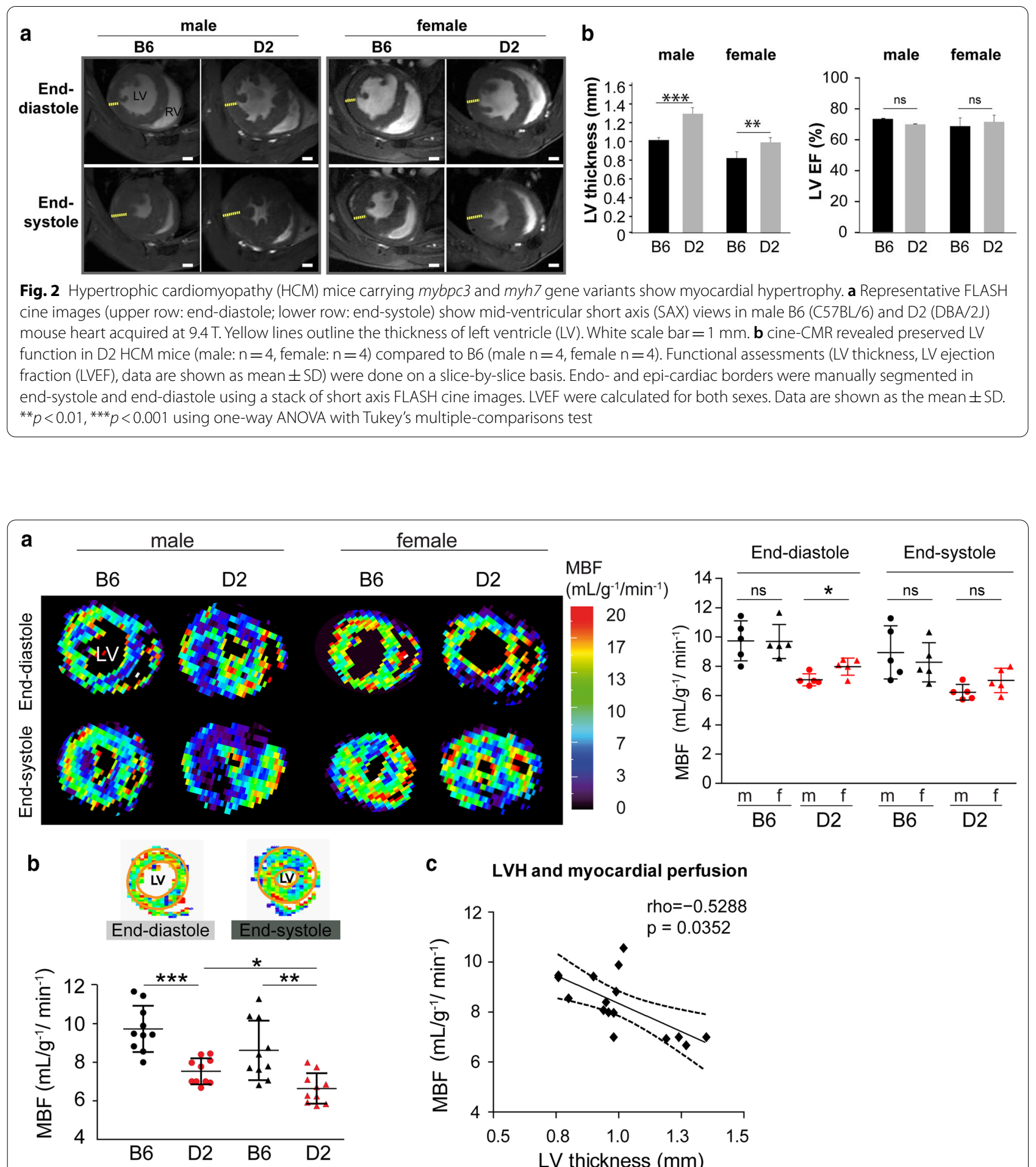

C LVH and myocardial perfusion

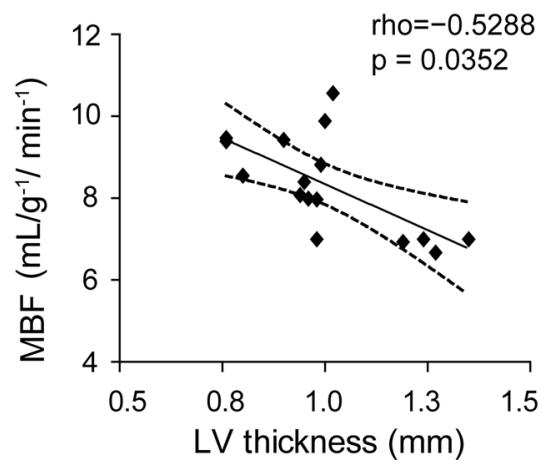

Fig. 3 Myocardial perfusion deficits in HCM mice. a Representative MBF maps showing end-diastolic (upper row) and end-systolic (lower row) cardiac phases for a mid-ventricular SAX views in both male and female control B6 and HCM (D2) mouse heart. MBF maps were acquired using cineALS CMR which facilitates the monitoring of dynamic MBF changes in the cardiac cycles. No sex difference in dynamic MBF changes in the cardiac cycles was observed between groups (B6 male $n=5, B 6$ female $n=5, D 2$ male $n=5$, D2 female $n=5$ ). Data are shown as the mean $\pm S D$. b Mean MBF were calculated for region of interest (ROI, depicted in orange colour by manually traced endocardial and epicardial contours) in mid-ventricular end-diastole and end-systole in all control (B6) and HCM (D2) mice ( $n=10$ for each group). Data are shown as the mean \pm SD. ${ }^{* *} p<0.01,{ }^{* * *} p<0.001$ using one-way ANOVA with Tukey's multiple-comparisons test. (c) Negative Spearman's rank correlations were seen between left ventricular thickness and myocardial blood flow $(n=16)$ 
resting global myocardial perfusion in the mid-ventricular slice was lower in D2 than in B6 mice (end-diastole: $\mathrm{D} 2 \mathrm{MBF}_{\text {global }}=7.5 \pm 0.6$ vs $\mathrm{B} 6 \mathrm{MBF}_{\text {global }}=9.3 \pm 1.6 \mathrm{ml} / \mathrm{g} /$ min, $p<0.05$; end-systole: $\mathrm{D} 2 \mathrm{MBF}_{\text {global }}=6.6 \pm 0.8$ vs $\mathrm{B} 6$ $\mathrm{MBF}_{\text {global }}=8.2 \pm 2.6 \mathrm{ml} / \mathrm{g} / \mathrm{min}, p<0.01$; Fig. 3b). Blood flow to the myocardium occurs mainly during diastole. We therefore compared the cyclic changes in blood flow in each group. In B6 control mice, the global myocardial perfusion did not differ between the end-systolic and end-diastolic phases $(p=0.090)$, whereas in D2 mice it was significantly lower in the end-systolic than in the end-diastolic phases $(p<0.05$; Fig. 3b).

Moreover, there were correlations between the global MBF and overall LV wall thickness. A significant negative correlation was observed between the MBF at rest and the correspondent LV thickness, i.e., thicker myocardium was associated with lower myocardial perfusion $($ rho $=-0.5288 ; p=0.0352$, non-parametric Spearman correlation; Fig. 3c).

\section{Resting myocardial perfusion deficit shows regional pattern}

Based on the segmentation model (Fig. 4a), we analysed the regional change of MBF. Septal myocardial perfusion was significantly lower in D2 than in B6 mice (end-diastole: D2 $\mathrm{MBF}_{\text {septal }}=7.9 \pm 1.0$ vs B6 $\mathrm{MBF}_{\text {septal }}=8.9 \pm 1.7 \mathrm{ml} / \mathrm{g} / \mathrm{min}, P<0.01$; end-systole: D2 $\mathrm{MBF}_{\text {septal }}=8.8 \pm 1.4$ vs B6 $\mathrm{MBF}_{\text {septal }}=6.9 \pm 1.5 \mathrm{ml} / \mathrm{g} /$ min, $p<0.05$; Fig. $4 \mathrm{~b})$. The inferior myocardial region adjacent to the septum showed lower MBF in D2 than in $\mathrm{B} 6$ mice (end-diastole: $\mathrm{D} 2 \mathrm{MBF}_{\text {inferior }}=9.1 \pm 2.2 \mathrm{vs} \mathrm{B6}$ $\mathrm{MBF}_{\text {inferior }}=6.4 \pm 0.5 \mathrm{ml} / \mathrm{g} / \mathrm{min}, p<0.05$; end-systole: $\mathrm{D} 2$ $\mathrm{MBF}_{\text {inferior }}=8.1 \pm 1.4$ vs $\mathrm{B} 6 \mathrm{MBF}_{\text {inferior }}=5.9 \pm 0.6 \mathrm{ml} / \mathrm{g} /$ min, $p<0.05)$. Lateral and anterior regions did not show significant MBF difference.

\section{Detection of impaired microvasculature and fibrosis in HCM mice}

The factors that might be involved in the reduction of small vessel function such as hypertrophy was determined (Figs. 2 and 3). We further confirmed the thickened LV and myofiber disarray by high resolution in vivo and ex vivo CMR in D2 mice (Fig. 5a). It is known that small vessels control local blood flow and impact the exchange functions of downstream segments of the myocardial microcirculation [21]. We therefore determined whether the small vessel density in the septal and inferior myocardium differ in those mice at steady state. Immunofluorescence staining for endothelial cells (with endothelial cell-specific Isolectin B4) revealed a lower number of Isolectin $\mathrm{B}^{+}{ }^{+}$vessels in D2 than in B6 mouse myocardium (D2 capillary $=31.0 \pm 3.8 \%$ vs B6 capillary $=40.7 \pm 4.6 \%, p<0.05$; Fig. $5 \mathrm{~b}$ ).

To visualize the myocardial fibrosis precisely, we performed collagen staining to indicate the exact position of fibrosis within the LV (Fig. 5c). Myocardial interstitial

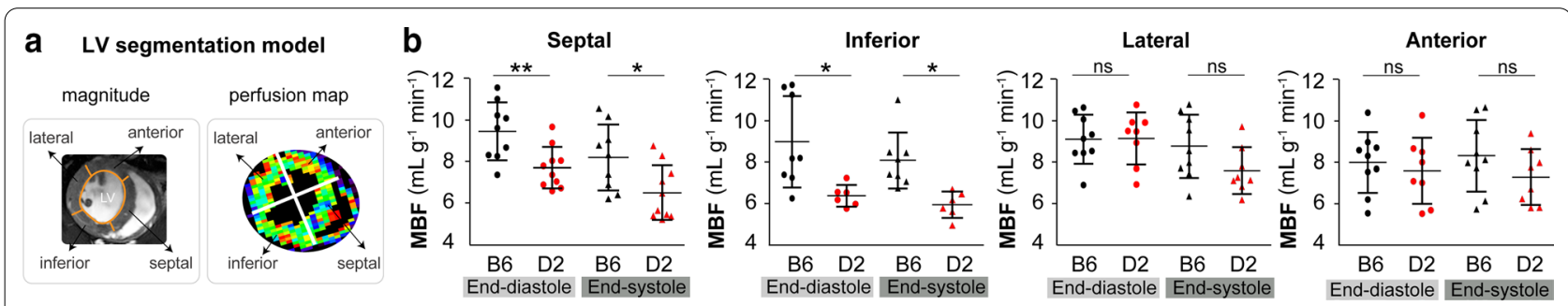

Fig. 4 Quantitative myocardial perfusion at rest. a Schematic illustration of the LV segmentation model for a mid-ventricular SAX slice of the mice heart. b Mean MBF were calculated for interventricular septum for a mid-ventricular slice during end-diastole and end-systole in all control (B6; $n=9)$ and $\mathrm{HCM}(\mathrm{D} 2 ; n=10)$ mice. Each dot represents an individual mouse. Data are shown as the mean $\pm S D .{ }^{*} p<0.05$, ${ }^{* *} p<0.01$, one-way ANOVA with Tukey's multiple-comparisons test

(See figure on next page.)

Fig. 5 a Representative long axis view of in vivo (in-plane spatial resolution $=57 \mu \mathrm{m}$ ) and ex vivo (in-plane spatial resolution $=30 \mu \mathrm{m}$ ) high resolution images showing detailed myocardial structure indicating hypertrophy. Right: A schematic to illustrate the relationship of hypertrophy, interstitial fibrosis, perivascular fibrosis and microvasculature. $\mathbf{b}$ Representative immunofluorescence staining of mouse heart LV sections for Isolectin B4 and the quantification of the capillary density ratio (number/5 imaging area). Scale bar $=50 \mu \mathrm{m}$; Data are shown as the mean \pm SD ( $n=3$ each sex groups; 6 B6 and 6 D2 mice, respectively). ${ }^{*} P<0.05$ ( $t$-test) c Sirius red staining show interstitial and perivascular collagen deposition. Upper row images scale bar $=500 \mu \mathrm{m}$; lower row images scale bar $=100 \mu \mathrm{m}$. Red arrow depicts perivascular fibrosis and blue arrow depicts interstitial fibrosis. Collagen volume fraction (CVF) and perivascular fibrosis ratio (PFR) in septal and inferior area of B6 $(n=8)$ and D2 $(n=8)$ mice left ventricle. Data are shown as the mean $\pm S D .{ }^{* *} p<0.01$, using $t$-test with Mann Whitney test 


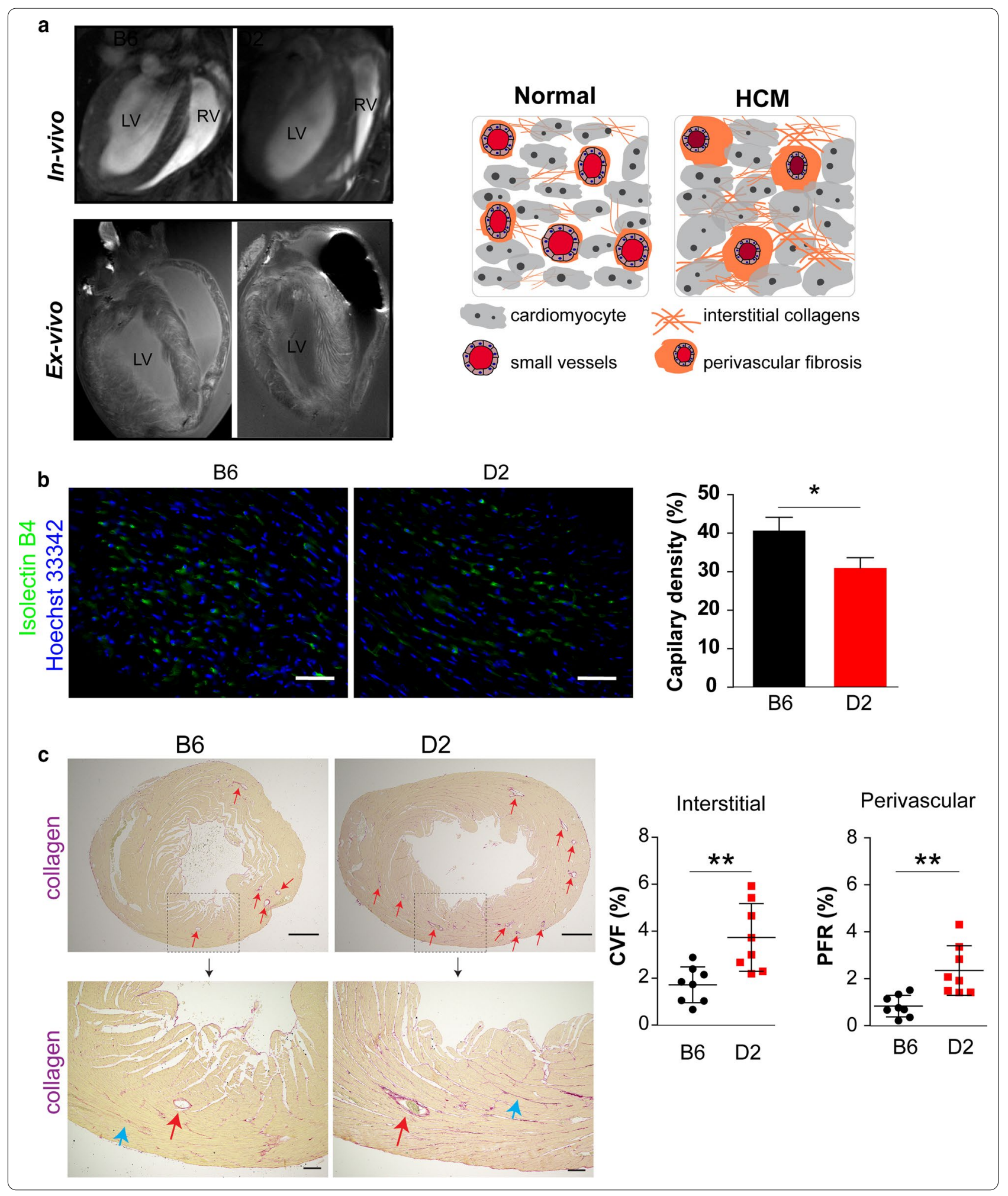


collagen was characterized by the increase in the percentage of total LV myocardial tissue occupied by collagen staining. CVF was significantly higher in D2 than B6 LV (D2 $\mathrm{CVF}=3.7 \pm 1.4 \%$ vs $\mathrm{B} 6 \mathrm{CVF}=1.7 \pm 0.7 \%$, $p<0.01)$. Furthermore, we also observed higher ratio of perivascular fibrosis in D2 mice versus B6 mice (D2 $\mathrm{PFR}=2.3 \pm 1.0 \%$, B6 PFR $=0.8 \pm 0.4 \%, p<0.01$ ). Microscopic characteristics revealed the relation of perfusion deficit with both perivascular and interstitial fibrosis.

\section{Discussion}

In this study, we demonstrate that cine ASL-CMR is capable of detecting changes in myocardial perfusion that are correlated to a reduction in small vessel density in the myocardium. The reduced myocardial perfusion provides an indication for microvascular dysfunction in HCM. The myocardial perfusion deficiency is correlated with $\mathrm{LVH}$ and associated with myocardial fibrosis and loss of small vessel densities, features which are presumably mediated by the genetic variants commonly found in HCM. We analysed the global as well as the regional differences of MBF based on the cardiac segmentation model. However, motion artefacts have been recognized as a limiting factor for the precise detection of myocardial regional differences. These artefacts can be induced by myocardial contraction/relaxation, by respiratory motion causing a shift a in the chest wall and the diaphragm, by susceptibility changes due to motion of the heart-lung or heart-liver interface or by non-uniform regionally motion patterns (different twist and strain) of the heart [35]. Another confounding factor could be the different rotational behaviour during early systole in mice [31]. The assessment of regional differences in myocardial perfusion pattern has to be further validated with respect to their functional component.

Subclinical myocardial remodelling dictates the progression of HCM, but currently diagnosis and treatment of the disease are restricted to measurements of myocardial hypertrophy. Diagnosing microvascular dysfunction has been a challenge. Standard tests used to diagnose coronary artery disease are not designed to detect small vessel disease, so a better understanding of the factors associated with microvascular dysfunction and its relation to the development of HCM would represent a major step toward earlier diagnosis and better management of the disease. Our results can partially explain previous findings showing that HCM patients have lower myocardial blood volume than healthy counterparts [22]. In the presence of $\mathrm{LVH}$, effective perfusion is critical to compensate for hypertrophy-induced micro-vessel restriction in the HCM heart. This hypothesis will need to be refined, tested and extended to obtain a fuller picture of the development of the pathological features of the disease and their consequences. In addition, ASL-CMR is not a standard clinical technique, while quantitative first-pass contrast agent based techniques are clinically established for myocardial perfusion imaging. Therefore, despite the necessity of bolus injections, robust quantitative first-pass post-processing methods are now becoming more widely available and currently represent the method of choice when similar studies are conducted in humans. En route to non-contrast myocardial perfusion imaging such as ASL-CMR, more studies are needed to prove that both methods are equally relevant for clinical applications [36].

Although a number of post-mortem studies have demonstrated marked impairment of the coronary microcirculation in the absence of significant coronary lesions in HCM patients [37, 38], the influence of genetic variants on microvascular function in HCM myocardium has remained unexplored. Measuring myocardial perfusion using CMR or other imaging modalities may reveal deficiencies in microvascular function even in mild cases or asymptomatic HCM patients, giving it potential as an independent predictor of clinical outcome [17, 39]. For instance, many patients with HCM have symptoms of myocardial ischemia and cardiac dysfunction. Abnormal intramural coronary arteries with markedly thickened walls and narrowed lumens are observed in HCM patients and may represent a genetic component of the underlying myocardial remodelling process [40]. Although the clinical relevance of microvascular dysfunction in HCM remains unclear, the fact that intramural coronary arteries exhibit structural alterations in areas of substantial myocardial fibrosis suggests a causal role for these arteries in producing ischemia [41]. In line with previous findings, we show in this study that the abnormal microvasculature was substantially pronounced in the inferior septum. Although it has become evident that perivascular fibrosis, but not interstitial fibrosis is associated with the impairment of coronary blood flow [42], we observed both perivascular and interstitial fibrosis are more pronounced in our HCM mouse model. Fibrosis is a dynamic process, thus the characterization of temporal pattern of both perivascular fibrosis and interstitial fibrosis requires additional attention [43].

Classically, HCM is characterized by varying degrees of LVH with a preserved or sometimes even increased LVEF [44]. Our data suggest that the quantification of cyclic changes of myocardial perfusion under resting conditions is sensitive to detect differences in HCM when using cineASL-based perfusion CMR. The incidence of heart failure (HF) in HCM patients is about 50\%, with symptoms varying from mild to severe $[45,46]$. Due to a substantial heterogeneity, ascertaining the incidence of which HF in HCM is challenging. In HCM, HF has two 
distinct clinical features: HFpEF or HFrEF. In the majority of HCM patients, HF is manifested as HFpEF phenotype, known as "diastolic heart failure" while only a minority develops HFrEF at a later stage. A recent large cohort study confirmed that systolic dysfunction (manifest by low LVEF) is highly associated with prognosis [47]. On the other hand, in HFpEF, microvascular dysfunction was evident as the major determinant of the pathological cascade that justifies clinical manifestations $[48,49]$. Therefore it is extremely relevant to identify microvascular dysfunction, including the cause and its mechanisms. Recent reports have suggested that patients with HFpEF exhibit an increased incidence of small vessel disease as shown by abnormal blood flow and increased microvascular resistance $[49,50]$. Our results support the idea that myocardial perfusion changes are connected to hypertrophy and the extent of fibrosis and thus can be an additional remodelling marker of the HCM phenotype regardless of cardiac dysfunction.

Blood flow through small vessels in the myocardium is influenced by changes in myocardial tissue pressure during heartbeats. Most myocardial perfusion occurs during the diastolic heart phase, when the myocardial pressure is low [51]. It may also be affected by total capillary density. Vascular endothelial dysfunction obviously impairs myocardial perfusion $[52,53]$; our study shows that capillary density is a potential key confounding factor for myocardial perfusion.

The accuracy of this non-invasive MBF measurement using cineASL has already been validated against the more common FAIR Look-Locker Gradient Echo technique, which was in turn validated against fluorescent microspheres [54]. The variations of blood flow throughout the cardiac cycle under different conditions were previously assessed using cineASL [30]. Notably, cyclic variation of MBF across the cardiac cycle has been thoroughly assessed using this technique in rats [24]. Since the MBF value in mice has been shown to be strongly dependent on the anesthetic conditions, the relatively high values found here could be due to differences in anesthesia. The anesthesia is also affected by the individual experimental conditions: actual isoflurane concentration inhaled, which is dependent on the face mask, space around the animal and gas recovery and therefore difficult to match across different lab setups. Other influencing factors are temperature and breathe rate. Therefore, one has to be cautious that the results on MBF may not reflect perfect resting conditions due to the vasodilating effect of isoflurane.

Sex differences are known in several facets of cardiac physiology [55]. Sex differences in myocardial perfusion have previously been demonstrated in healthy subjects and in patients with nonischemic heart failure using positron emission tomography (PET) or CMR [56, 57]. These reports suggested that females have higher resting myocardial perfusion compared to males. Our data showed the tendency of higher resting MBF in both diastolic and systolic cardiac phases. The sex difference of myocardial perfusion will therefore need to be takin into account in future studies.

\section{Limitations}

While we observed the sex differences in end-diastolic MBF in D2 mice but not in other experimental groups, this observation might be attributed to the small sample size used, which is a recognized limitation of our study. We are therefore unable to comment on the exact sex influence that might exist with perfusion abnormalities. To address this scientific question future work will need to incorporate a larger sample size. The cardiac function and MBF measurements were not derived from the same group of mice. Notably, the physiological effects of anaesthesia during CMR have to be minimized. Consequently, we have designed the CMR measurement protocol such that the total duration of an individual in vivo experiment does not exceed a maximum of $60 \mathrm{~min}$. Therefore all measurements were performed in the same time window.

We noted that MBF values in mice found in this study are higher when compared with published findings [30, $58,59]$. Notwithstanding our findings remain comparable and within the error ranges given in the literature [60]. Multiple reasons can contribute to modifications of the resting perfusion in rodents such as the level of anesthesia, gas mixture or temperature. The comparatively high control values have also been obtained by Abdesselam et al. [58], who attributed this to a moderate cardiac stress condition due to isoflurane-dependent vasodilation to the elevated baseline values. In previous studies in healthy mice using ASL-CMR reported myocardial perfusion ranging from $5.0 \pm 0.8$ to $6.9 \pm 1.7 \mathrm{ml} / \mathrm{g} / \mathrm{min}[30$, $59,61]$ at $4.7 \mathrm{~T}$ and $7.0 \pm 0.5 \mathrm{ml} / \mathrm{g} / \mathrm{min}$ at $7 \mathrm{~T} \mathrm{[60].} \mathrm{Our}$ experiments were performed at $9.4 \mathrm{~T}$ and revealed an average MBF of $8.2 \pm 2.6 \mathrm{ml} / \mathrm{g} / \mathrm{min}$, which is in accordance with the literature values. No specific validation against gold-standard techniques has been done for perfusion measured in this study. However, the employed techniques were earlier validated in rats but not mice.

Our study did not tackle the influence of single genetic mutations. Therefore, the specific effects of Mybpc3 or Myh7 point mutations need to be pursued, as does the relation between oxygen consumption and myocardial perfusion in HCM.

To tackle the diastolic dysfunction, we did not include myocardial strain measurements in our study because we would like to first emphasize the early microstructural change such as vascular deficiency in HCM. MR 
tagging can be useful for detecting changes in myocardial strain in the mouse heart. We anticipate including this approach into our future studies. Our preliminary results provide the basis for future investigations of the entire disease course, including the early disease phase when the pathological changes are subtle. Then by performing a comprehensive protocol of CMR measurements including parametric mapping $\left(\mathrm{T}_{1}, \mathrm{~T}_{2}\right.$ and $\left.\mathrm{T}_{2}{ }^{*}\right)$ and ASL, we can observe how CMR biomarkers change over time, and how these changes can predict later disease outcomes.

\section{Conclusions}

We used state-of-the-art in vivo CMR to evaluate microstructure and functional changes in mice with $\mathrm{HCM}$ genetic variants. We have demonstrated that small vessel dysfunction is present in these HCM variants in association with myocardial fibrosis. Our work thus suggests that more research on MBF as an imaging marker with a potential to monitor early myocardial microvascular remodelling is relevant in the field.

\begin{abstract}
Abbreviations
ASL: Arterial spin labelling; Bvf: Fractional tissue blood volume per cardiac tissue volume; CMR: Cardiovascular magnetic resonance; Cine ASL-CMR: Dynamic arterial spin labelling based CMR; CVF: Collagen volume fraction; ECG: Electrocardiogram; FLASH: Fast low angle shot; FOV: Field-of-view; HCM Hypertrophic cardiomyopathy; HFpEF: Heart failure with preserved ejection fraction; HFrEF: Heart failure with reduced ejection fraction; LV: Left ventricle/ left ventricular; LVEF: Left ventricular ejection fraction; LVH: Left ventricular hypertrophy; MBF: Myocardial blood flow; MYBPC3: Cardiac myosin-binding protein C; MYH7: $\beta-$ Myosin-heavy chain; PFR: Perivascular fibrosis ratio; SAx: Short axis; TR: Repetition time; TE: Echo time; SD: Standard deviation.
\end{abstract}

\section{Acknowledgements}

The Helmholtz Association supports open access based upon the "Berlin Declaration on Open Access to Knowledge in the Sciences and Humanities". The authors wish to thank Bernadette Nickl, Thomas Rathjen, Petya Jordan and Elena Cano Rincon and Russell Hodge for reagents, fruitful discussions and intellectual input.

\section{Authors' contributions}

All authors contributed to the study conception and design. Material preparation, data collection and analysis were performed by MCK, YCL and FQ. FK, AP, MB, LC and TN have jointly supervised the work. The first draft of the manuscript was written by MCK and all authors commented on previous versions of the manuscript. All authors read and approved the final manuscript.

\section{Funding}

Open Access funding enabled and organized by Projekt DEAL. This work was funded in part by the German Centre for Cardiovascular Research (DZHK; BER 6.1, partner site Berlin) and by the Federal Ministry of Education and Research, Berlin, Germany, FKZ 81 Z6100161.

\section{Availability of data and materials}

The data derived from ASL-CMR are public and available under https://figsh are.com/articles/figure/IDL_images/14251241. Our group is also initiator of Open Source MRI (http://www.opensourceimaging.org/). R script for statistical analysis of 4 myocardial regions is published on the GitHub (https://doi.org/ 10.5281/zenodo.4061597). Experimental design and data collection were handled according to the ARRIVE guidelines (https://www.nc3rs.org.uk/sites/ default/files/documents/Guidelines/NC3Rs\%20ARRIVE\%20Guidelines\%20Che cklist\%20\%28fillable\%29.pdf).

\section{Declarations}

Ethics approval and consent to participate

All studies were approved by the Berlin State review board at the "Landesamt für Gesundheit und Soziales (LAGeSo)". Mice were handled according to the LAGeSo and internal (MDC) rules and regulations.

\section{Consent for publication}

Not applicable.

\section{Competing interests}

The authors declare no conflicting interests in connection with this manuscript.

\section{Author details}

${ }^{1}$ Berlin Ultrahigh Field Facility (B.U.F.F.), Max Delbrück Center for Molecular Medicine in the Helmholtz Association (MDC), Robert-Rössle Strasse 10, 13125 Berlin, Germany. ${ }^{2}$ DZHK (German Centre for Cardiovascular Research), Partner site Berlin, Berlin, Germany. ${ }^{3}$ Centre de Résonance Magnétique Biologique et Médicale (CRMBM), Aix-Marseille University, CNRS, Marseille, France. ${ }^{4}$ Molecular Biology of Peptide Hormones, Max Delbrück Center for Molecular Medicine in the Helmholtz Association (MDC), Berlin, Germany. ${ }^{5}$ Department of Experimental Pharmacology and Toxicology, University Medical Center Hamburg-Eppendorf, Hamburg, Germany. ${ }^{6}$ DZHK (German Centre for Cardiovascular Research), Partner site Hamburg/Kiel/Lübeck, Berlin, Germany. ${ }^{7}$ Experimental and Clinical Research Center (ECRC), A Joint Cooperation between the Charité Medical Faculty and the Max-Delbrück Center for Molecular Medicine, Berlin, Germany.

Received: 6 January 2020 Accepted: 6 April 2021

Published online: 31 May 2021

\section{References}

1. Semsarian C, Ingles J, Maron MS, Maron BJ. New perspectives on the prevalence of hypertrophic cardiomyopathy. J Am Coll Cardiol. 2015;65(12):1249-54.

2. Franz WM, Muller OJ, Katus HA. Cardiomyopathies: from genetics to the prospect of treatment. Lancet. 2001;358(9293):1627-37.

3. Bagnall RD, Weintraub RG, Ingles J, Duflou J, Yeates L, Lam L, Davis AM, Thompson T, Connell V, Wallace J, et al. A prospective study of sudden cardiac death among children and young adults. N Engl J Med. 2016:374(25):2441-52.

4. Maron BJ, Doerer JJ, Haas TS, Tierney DM, Mueller FO. Sudden deaths in young competitive athletes: analysis of 1866 deaths in the United States, 1980-2006. Circulation. 2009;119(8):1085-92.

5. Michels M, Soliman Ol, Phefferkorn J, Hoedemaekers YM, Kofflard MJ, Dooijes D, Majoor-Krakauer D, Ten Cate FJ. Disease penetrance and risk stratification for sudden cardiac death in asymptomatic hypertrophic cardiomyopathy mutation carriers. Eur Heart J. 2009;30(21):2593-8.

6. Maron BJ, Maron MS. Hypertrophic cardiomyopathy. Lancet. 2013;381(9862):242-55.

7. Ingles J, Goldstein J, Thaxton C, Caleshu C, Corty EW, Crowley SB, Dougherty K, Harrison SM, McGlaughon J, Milko LV, et al. Evaluating the clinical validity of hypertrophic cardiomyopathy genes. Circ Genom Precis Med. 2019;12(2):e002460

8. Toepfer CN, Wakimoto H, Garfinkel AC, McDonough B, Liao D, Jiang J, Tai AC, Gorham JM, Lunde IG, Lun M, et al. Hypertrophic cardiomyopathy mutations in MYBPC3 dysregulate myosin. Sci Transl Med. 2019. https:// doi.org/10.1126/scitranslmed.aat1199.

9. Maron BJ, Ommen SR, Semsarian C, Spirito P, Olivotto I, Maron MS. Hypertrophic cardiomyopathy: present and future, with translation into contemporary cardiovascular medicine. J Am Coll Cardiol. 2014;64(1):83-99.

10. Burchfield JS, Xie M, Hill JA. Pathological ventricular remodeling: mechanisms: part 1 of 2. Circulation. 2013;128(4):388-400.

11. Hayashi M, Shimizu W, Albert CM. The spectrum of epidemiology underlying sudden cardiac death. Circ Res. 2015;116(12):1887-906. 
12. Weissler-Snir A, Adler A, Williams L, Gruner C, Rakowski H. Prevention of sudden death in hypertrophic cardiomyopathy: bridging the gaps in knowledge. Eur Heart J. 2017;38(22):1728-37.

13. Maron BJ. Clinical course and management of hypertrophic cardiomyopathy. N Engl J Med. 2018;379(7):655-68.

14. Soler R, Mendez C, Rodriguez E, Barriales R, Ochoa JP, Monserrat L. Phenotypes of hypertrophic cardiomyopathy. An illustrative review of MRI findings. Insights Imaging. 2018;9(6):1007-20.

15. Raman B, Ariga R, Spartera M, Sivalokanathan S, Chan K, Dass S, Petersen SE, Daniels MJ, Francis J, Smillie R, et al. Progression of myocardial fibrosis in hypertrophic cardiomyopathy: mechanisms and clinical implications. Eur Heart J Cardiovasc Imaging. 2019;20(2):157-67.

16. Petersen SE, Jerosch-Herold M, Hudsmith LE, Robson MD, Francis JM, Doll HA, Selvanayagam JB, Neubauer S, Watkins H. Evidence for microvascular dysfunction in hypertrophic cardiomyopathy: new insights from multiparametric magnetic resonance imaging. Circulation. 2007;115(18):2418-25.

17. Cecchi F, Olivotto I, Gistri R, Lorenzoni R, Chiriatti G, Camici PG. Coronary microvascular dysfunction and prognosis in hypertrophic cardiomyopathy. N Engl J Med. 2003;349(11):1027-35

18. Timmer SA, Knaapen P. Coronary microvascular function, myocardial metabolism, and energetics in hypertrophic cardiomyopathy: insights from positron emission tomography. Eur Heart J Cardiovasc Imaging. 2013;14(2):95-101.

19. Rosen BD, Lima JA, Nasir K, Edvardsen T, Folsom AR, Lai S, Bluemke DA, Jerosch-Herold M. Lower myocardial perfusion reserve is associated with decreased regional left ventricular function in asymptomatic participants of the multi-ethnic study of atherosclerosis. Circulation. 2006:114(4):289-97.

20. Duncker DJ, Koller A, Merkus D, Canty JM J.r. Regulation of coronary blood flow in health and ischemic heart disease. Prog Cardiovasc Dis. 2015;57(5):409-22.

21. Jacob M, Chappell D, Becker BF. Regulation of blood flow and volume exchange across the microcirculation. Crit Care. 2016;20(1):319.

22. Huelnhagen T, Ku MC, Reimann HM, Serradas Duarte T, Pohlmann A, Flemming B, Seeliger E, Eichhorn C, Ferrari VA, Prothmann M, et al. Myocardial effective transverse relaxation time $T 2^{*}$ ) is elevated in hypertrophic cardiomyopathy: A 7.0 T magnetic resonance imaging study. Sci Rep. 2018;8(1):3974

23. Huelnhagen T, Hezel F, Serradas Duarte T, Pohlmann A, Oezerdem C, Flemming B, Seeliger E, Prothmann M, Schulz-Menger J, NiendorfT. Myocardial effective transverse relaxation time $\mathrm{T}^{*}$ Correlates with left ventricular wall thickness: a 7.0 T MRI study. Magn Reson Med. 2017;77(6):2381-9.

24. Troalen T, Capron T, Bernard M, Kober F. In vivo characterization of rodent cyclic myocardial perfusion variation at rest and during adenosineinduced stress using cine-ASL cardiovascular magnetic resonance. J Cardiovasc Magn Reson. 2014;16:18.

25. Kober F, Jao T, Troalen T, Nayak KS. Myocardial arterial spin labeling. J Cardiovasc Magn Reson. 2016;18:22.

26. Naresh NK, Chen X, Moran E, Tian Y, French BA, Epstein FH. Repeatability and variability of myocardial perfusion imaging techniques in mice: Comparison of arterial spin labeling and first-pass contrast-enhanced MRI. Magn Reson Med. 2016;75(6):2394-405.

27. Thomson KL, Ormondroyd E, Harper AR, Dent T, McGuire K, Baksi J, Blair E, Brennan P, Buchan R, Bueser T, et al. Analysis of 51 proposed hypertrophic cardiomyopathy genes from genome sequencing data in sarcomere negative cases has negligible diagnostic yield. Genet Med. 2019;21(7):1576-84

28. Zhao W, Zhao T, Chen Y, Zhao F, Gu Q, Williams RW, Bhattacharya SK, Lu L, Sun Y. A Murine Hypertrophic Cardiomyopathy Model: The DBA/2J Strain. PLOS ONE. 2015;10(8):e0133132.

29. Ku MC, Huelnhagen T, Niendorf T, PohImann A. Cardiac MRI in Small Animals. Methods Mol Biol. 2018:1718:269-84.

30. Troalen T, Capron T, Cozzone PJ, Bernard M, Kober F. Cine-ASL: a steadypulsed arterial spin labeling method for myocardial perfusion mapping in mice. Part I. Experimental study. Magn Reson Med. 2013;70(5):1389-98.

31. Jung B, Odening KE, Dall'Armellina E, Foll D, Menza M, MarkI M, Schneider JE. A quantitative comparison of regional myocardial motion in mice, rabbits and humans using in-vivo phase contrast CMR. J Cardiovasc Magn Reson. 2012:14:87.
32. Ide S, Riesenkampff E, Chiasson DA, Dipchand Al, Kantor PF, Chaturvedi RR, Yoo SJ, Grosse-Wortmann L. Histological validation of cardiovascular magnetic resonance $\mathrm{T1}$ mapping markers of myocardial fibrosis in paediatric heart transplant recipients. J Cardiovasc Magn Reson. 2017;19(1):10.

33. Rikitake Y, Oyama N, Wang CY, Noma K, Satoh M, Kim HH, Liao JK. Decreased perivascular fibrosis but not cardiac hypertrophy in ROCK1+/haploinsufficient mice. Circulation. 2005;112(19):2959-65.

34. Morita H, Rehm HL, Menesses A, McDonough B, Roberts AE, Kucherlapati R, Towbin JA, Seidman JG, Seidman CE. Shared genetic causes of cardiac hypertrophy in children and adults. N Engl J Med. 2008;358(18):1899-908.

35. Del-Canto I, Lopez-Lereu MP, Monmeneu JV, Croisille P, Clarysse P, Chorro FJ, Bodi V, Moratal D. Characterization of normal regional myocardial function by MRI cardiac tagging. J Magn Reson Imaging. 2015;41(1):83-92.

36. Do HP, Ramanan V, Qi X, Barry J, Wright GA, Ghugre NR, Nayak KS. Noncontrast assessment of microvascular integrity using arterial spin labeled cardiovascular magnetic resonance in a porcine model of acute myocardial infarction. J Cardiovasc Magn Reson. 2018;20(1):45.

37. Basso C, Thiene G, Corrado D, Buja G, Melacini P, Nava A. Hypertrophic cardiomyopathy and sudden death in the young: pathologic evidence of myocardial ischemia. Hum Pathol. 2000;31(8):988-98.

38. Galati G, Leone O, Pasquale F, Olivotto I, Biagini E, Grigioni F, Pilato E, Lorenzini M, Corti B, Foa A, et al. Histological and histometric characterization of myocardial fibrosis in end-stage hypertrophic cardiomyopathy: a clinical-pathological study of 30 explanted hearts. Circ Heart Fail. 2016. https://doi.org/10.1161/CIRCHEARTFAILURE.116.003090.

39. Villemain O, Correia M, Mousseaux E, Baranger J, Zarka S, Podetti I, Soulat G, Damy T, Hagege A, Tanter M, et al. Myocardial stiffness evaluation using noninvasive shear wave imaging in healthy and hypertrophic cardiomyopathic adults. JACC Cardiovasc Imaging. 2019;12(7 Pt 1):1135-45.

40. Maron BJ, Wolfson JK, Epstein SE, Roberts WC. Intramural ("small vessel") coronary artery disease in hypertrophic cardiomyopathy. J Am Coll Cardiol. 1986;8(3):545-57.

41. Camaioni C, Knott KD, Augusto JB, Seraphim A, Rosmini S, Ricci F, Boubertakh R, Xue H, Hughes R, Captur G, et al. Inline perfusion mapping provides insights into the disease mechanism in hypertrophic cardiomyopathy. Heart. 2020;106(11):824-9.

42. Dai Z, Aoki T, Fukumoto Y, Shimokawa H. Coronary perivascular fibrosis is associated with impairment of coronary blood flow in patients with nonischemic heart failure. J Cardiol. 2012;60(5):416-21.

43. de Boer RA, De Keulenaer G, Bauersachs J, Brutsaert D, Cleland JG, Diez J, Du XJ, Ford P, Heinzel FR, Lipson KE, et al. Towards better definition, quantification and treatment of fibrosis in heart failure. A scientific roadmap by the Committee of Translational Research of the Heart Failure Association (HFA) of the European Society of Cardiology. Eur J Heart Fail. 2019;21(3):272-85.

44. Lam CSP, Voors AA, de Boer RA, Solomon SD, van Veldhuisen DJ. Heart failure with preserved ejection fraction: from mechanisms to therapies. Eur Heart J. 2018;39(30):2780-92.

45. Seferovic PM, Polovina M, Bauersachs J, Arad M, Gal TB, Lund LH, Felix SB, Arbustini E, Caforio ALP, Farmakis D, et al. Heart failure in cardiomyopathies: a position paper from the Heart Failure Association of the European Society of Cardiology. Eur J Heart Fail. 2019;21(5):553-76.

46. Maron BJ, Rowin EJ, Casey SA, Link MS, Lesser JR, Chan RH, Garberich RF, Udelson JE, Maron MS. Hypertrophic cardiomyopathy in adulthood associated with low cardiovascular mortality with contemporary management strategies. J Am Coll Cardiol. 2015;65(18):1915-28.

47. Marstrand P, Han L, Day SM, Olivotto I, Ashley EA, Michels M, Pereira AC, Wittekind SG, Helms A, Saberi S, et al. Hypertrophic cardiomyopathy with left ventricular systolic dysfunction: insights from the SHaRe registry. Circulation. 2020;141(17):1371-83.

48. Crea F, Bairey Merz CN, Beltrame JF, Kaski JC, Ogawa H, Ong P, Sechtem U, Shimokawa H, Camici PG, Coronary Vasomotion Disorders International Study Group. The parallel tales of microvascular angina and heart failure with preserved ejection fraction: a paradigm shift. Eur Heart J. 2017;38(7):473-7.

49. D'Amario D, Migliaro S, Borovac JA, Restivo A, Vergallo R, Galli M, Leone AM, Montone RA, Niccoli G, Aspromonte N, et al. Microvascular dysfunction in heart failure with preserved ejection fraction. Front Physiol. 2019;10:1347.

50. Joung S, Wei J, Nelson MD, Aldiwani H, Shufelt C, Tamarappoo B, Berman D, Thomson LEJ, Bairey Merz CN. Progression of coronary microvascular 
dysfunction to heart failure with preserved ejection fraction: a case report. J Med Case Rep. 2019;13(1):134.

51. Goodwill AG, Dick GM, Kiel AM, Tune JD. Regulation of coronary blood flow. Compr Physiol. 2017;7(2):321-82.

52. Marti CN, Gheorghiade M, Kalogeropoulos AP, Georgiopoulou W, Quyyumi AA, Butler J. Endothelial dysfunction, arterial stiffness, and heart failure. J Am Coll Cardiol. 2012:60(16):1455-69.

53. Fernlund E, Gyllenhammar T, Jablonowski R, Carlsson M, Larsson A, Arnlov J, Liuba P. Serum biomarkers of myocardial remodeling and coronary dysfunction in early stages of hypertrophic cardiomyopathy in the young. Pediatr Cardiol. 2017;38(4):853-63.

54. Jacquier A, Kober F, Bun S, Giorgi R, Cozzone PJ, Bernard M. Quantification of myocardial blood flow and flow reserve in rats using arterial spin labeling MRI: comparison with a fluorescent microsphere technique. NMR Biomed. 2011;24(9):1047-53.

55. Mauvais-Jarvis F, Bairey Merz N, Barnes PJ, Brinton RD, Carrero JJ, DeMeo DL, De Vries GJ, Epperson CN, Govindan R, Klein SL, et al. Sex and gender: modifiers of health, disease, and medicine. Lancet. 2020;396(10250):565-82.

56. Kadkhodayan A, Lin CH, Coggan AR, Kisrieva-Ware Z, Schechtman KB, Novak E, Joseph SM, Davila-Roman VG, Gropler RJ, Dence $C$, et al. Sex affects myocardial blood flow and fatty acid substrate metabolism in humans with nonischemic heart failure. J Nucl Cardiol. 2017;24(4):1226-35.

57. Nickander J, Themudo R, Sigfridsson A, Xue H, Kellman P, Ugander M. Females have higher myocardial perfusion, blood volume and extracellular volume compared to males-an adenosine stress cardiovascular magnetic resonance study. Sci Rep. 2020;10(1):10380.
58. Abdesselam I, Pepino P, Troalen T, Macia M, Ancel P, Masi B, Fourny N, Gaborit B, Giannesini B, Kober F, et al. Time course of cardiometabolic alterations in a high fat high sucrose diet mice model and improvement after GLP-1 analog treatment using multimodal cardiovascular magnetic resonance. J Cardiovasc Magn Reson. 2015;17:95.

59. Vandsburger MH, French BA, Helm PA, Roy RJ, Kramer CM, Young AA, Epstein FH. Multi-parameter in vivo cardiac magnetic resonance imaging demonstrates normal perfusion reserve despite severely attenuated beta-adrenergic functional response in neuronal nitric oxide synthase knockout mice. Eur Heart J. 2007;28(22):2792-8.

60. Streif JU, Nahrendorf M, Hiller KH, Waller C, Wiesmann F, Rommel E, Haase A, Bauer WR. In vivo assessment of absolute perfusion and intracapillary blood volume in the murine myocardium by spin labeling magnetic resonance imaging. Magn Reson Med. 2005;53(3):584-92.

61. Kober F, Iltis I, Cozzone PJ, Bernard M. Myocardial blood flow mapping in mice using high-resolution spin labeling magnetic resonance imaging: influence of ketamine/xylazine and isoflurane anesthesia. Magn Reson Med. 2005;53(3):601-6.

\section{Publisher's Note}

Springer Nature remains neutral with regard to jurisdictional claims in published maps and institutional affiliations.
Ready to submit your research? Choose BMC and benefit from:

- fast, convenient online submission

- thorough peer review by experienced researchers in your field

- rapid publication on acceptance

- support for research data, including large and complex data types

- gold Open Access which fosters wider collaboration and increased citations

- maximum visibility for your research: over 100M website views per year

At BMC, research is always in progress.

Learn more biomedcentral.com/submissions 